\title{
Simulation of liquid composite moulding using a finite volume scheme and the level- set method
}

Sandberg, Michael; Hattel, Jesper H.; Spangenberg, Jon

Published in:

International Journal of Multiphase Flow

Link to article, DOI:

10.1016/j.ijmultiphaseflow.2019.06.014

Publication date:

2019

Document Version

Peer reviewed version

Link back to DTU Orbit

Citation (APA):

Sandberg, M., Hattel, J. H., \& Spangenberg, J. (2019). Simulation of liquid composite moulding using a finite volume scheme and the level-set method. International Journal of Multiphase Flow, 118, 183-192.

https://doi.org/10.1016/j.ijmultiphaseflow.2019.06.014

\section{General rights}

Copyright and moral rights for the publications made accessible in the public portal are retained by the authors and/or other copyright owners and it is a condition of accessing publications that users recognise and abide by the legal requirements associated with these rights.

- Users may download and print one copy of any publication from the public portal for the purpose of private study or research.

- You may not further distribute the material or use it for any profit-making activity or commercial gain

- You may freely distribute the URL identifying the publication in the public portal 


\section{Simulation of liquid composite moulding using a finite volume scheme and}

In this paper, a 2D numerical framework for the simulation of flows in porous media that are characterised by a sharp transition between the saturated and unsaturated zone is presented. Using a finite volume scheme and the level-set method, the framework is derived based on a conventional, implicit solution of Darcy's law for the pressure field, while the level-set function is advected explicitly locally at the flow front. With the main application to liquid composite moulding (LCM) in mind, the numerical framework is verified against analytical solutions, experiments, and other benchmark cases for a variety of situations that occur in this composite manufacturing process. The cases include local changes in permeability related to edge-effects, merging of flow fronts, and continuous manufacturing processes. It is highlighted that the numerical framework achieves convergence with a spatial accuracy between $1^{\text {st }}$ and $2^{\text {nd }}$-order; the level-set operations only take about $20 \%$ of the total CPU time; and the propagation of the resin front can be achieved with CFL-sized time steps without overfilling cells and without applying any artificial smoothing.

Keywords: Resin-impregnation; Liquid composite moulding; Darcy flow; Finite-volume method; Level-set

\section{Introduction}

Fibre-reinforced polymer composites (FRPC) can be manufactured using a variety of liquid composite moulding (LCM) techniques. Among these manufacturing technologies exist e.g. vacuum infusion moulding, resin-transfer moulding, compression moulding, and resin-injection pultrusion. While the latter process deviates from the other by being a continuous process, the different sub-steps in the process cycle are the same. First, the dry fibre material is placed in a mould and the mould is secured. The fibre material is then impregnated with a polymer (the resin), and the part is left to cure. Finally, the cured part is demoulded and the process can be repeated.

${ }^{*}$ Corresponding author

Email addresses: misan@mek.dtu.dk (Michael Sandberg), jhat@mek.dtu.dk (Jesper H. Hattel), josp@mek.dtu.dk (Jon Spangenberg) 


\begin{tabular}{|c|c|c|c|}
\hline \multicolumn{4}{|c|}{ Nomenclature } \\
\hline$\alpha$ & Liquid volume fraction of cell & $n$ & Time step \\
\hline$a, b, c$ & Line coefficients & $\mathbf{n}$ & Surface normal \\
\hline$\beta, \chi$ & Wendland function and parameter & $\Omega, \Gamma$ & Area and contour domain \\
\hline$d$ & Distance to interface & $p$ & Pressure \\
\hline $\bar{d}$ & Distance to interface, approximation & $\phi$ & Level-set function \\
\hline$\Delta x, \Delta y$ & Grid spacing in $x$ - and $y$-directions & q & Superficial velocity \\
\hline$H$ & Window function & $r_{f}$ & Flow front radius \\
\hline$h$ & Distance to interface normal & $S$ & Volume of injected resin \\
\hline K & Permeability tensor & & \\
\hline$K^{\prime}$ & Quasi-isotropic permeability & $s$ & Source or sink term \\
\hline$L()$ & Differential operator & $\psi$ & Degree of anisotropy \\
\hline$L, w$ & Length and width & $\theta_{v}$ & Porosity \\
\hline$L_{2}, L_{\infty}$ & Error norms & $t, \tau$ & Time, pseudo time \\
\hline$\mu$ & Viscosity & $\mathbf{u}$ & Velocity \\
\hline$N$ & Number of cells & $x, y, z$ & Spatial coordinates \\
\hline
\end{tabular}

Current research related to simulation of the impregnation step in LCM generally follows one of two concepts: Either the flow is considered to be saturated or unsaturated. Saturated flow implies that there exists a sharp transition between the resin (the saturated zone) and the dry fibre-material (the unsaturated zone) during the impregnation step. Unsaturated flow, on the other hand, includes the transient, progressive impregnation mechanisms by considering a partially saturated zone as part of the impregnation step. Although saturated flow is the focus of this paper, we briefly elaborate on unsaturated flow in order to put simulation of saturated flow into perspective.

One method for simulation of unsaturated flow is to consider the governing phenomena to be dual-phased. In this method, the first phase is a saturated flow in the pore space between tows (rovings of fibres), while the second phase concerns the flow of resin from the pore space into the tows, see e.g. Binétruy et al. [4], DeParseval et al. [11], Pillai [29], and Patiño-Arcila and Vanegas-Jaramillo [28]. This approach is easy to relate to the flow behaviour observed in impregnation experiments of e.g. woven or non-crimp fabrics [30]. Such fabrics have a bimodal distribution of tows and pore space that generally facilitates dual-phase flow [23]. Unsaturated flow has 
also been described by methodologies adopted from soil science. The progressive saturation in this approach is related to the level of pressure and the relative permeability, through a semi-empirical expression of the saturation behaviour, see e.g. Nordlund and Michaud [25] and Michaud [23].

Common for the two approaches above is that the saturation behaviour enters through a nonlinearity in the mass balance equation, which in certain cases can be problematic. For example, when the partially saturated zone is short, e.g. in layups with limited pore space and a more homogeneous fibre distribution (CSM or CFM mats, or layups of tows/rovings in pultrusion processes), the nonlinearity becomes strong, as saturation occurs rapidly. A strong nonlinearity is undesirable for any numerical framework, as it increases the computational effort to achieve convergence. In these cases, it can be more appropriate to consider the flow as saturated, i.e. when the partially saturated zone is short in comparison to other relevant dimensions.

There exist multiple solutions methods for the simulation of saturated flow in LCM, but common for all methods is that a numerical scheme is needed to advance the resin front $[5,38,24,34,36,39,37,32]$. Initially developed in the '90s by Bruschke and Advani [5] and Trochu et al. [38], the finite element/control volume (FE/CV) method is still the most popular method for simulation of LCM [28]. Varying in detail, the overall approach of the method is to apply the finite element method for the solution of the pressure field, while the impregnated zone develops through the filling of CVs. If overfilling of the CVs is to be avoided, the time step is constrained by the step-size that results in complete filling of, at least, one control volume. The FE/CV method is the basis for the commercial software packages PAM-RTM, RTM-Worx, and LIMS, and has been applied to a variety of applications related to LCM. For example, non-isothermal flows [6], inlet gate control and dry-spot detection [20], edge-effects in LCM [3], compression moulding [2], fibre compaction [1], and more.

As an alternative to the FE/CV-method, general Eulerian free surface techniques specify the flow front location and transport the flow front itself. This allows for an accurate representation of the flow front location and relaxes the threshold on the allowed time-step size. Explicit free surface solvers are generally constrained by the Courant-Friedrichs-Lewy (CFL) condition, while fully implicit free surface solvers can in principle be unconditionally stable. Today, most free-surface techniques are based on the volume of fluid method [18, 7] or the level-set method [27], where the latter is the focus of this paper.

In the context of LCM, Soukane and Trochu [37] were the first to introduce the level-set method. They combined a level-set and the boundary element method to simulate LCM on general unstructured meshes adapted to the flow front location. In Soukane and Trochu's work, the flow front location was represented by a signed distance function and tracked using a fast-marching method. The level-set method has also been applied in a finite element framework by Jung et al. [19]. In their work, elements cut by the flow front were enriched using the extended finite element method (XFEM) to handle the change of material properties in the vicinity of the flow front. While XFEM is a method for resolving discontinuities, the numerical framework in [19] still required to smear out the resin viscosity near the resin front.

In this work, we present a numerical framework for the simulation of LCM based on a finite volume (FV) 
scheme and the level-set method. The framework is based on a conventional, implicit solution of Darcy's law for the pressure field, while the level-set function is advected explicitly locally at the resin flow front. Compared to the previous work in the field, the framework does not need to smear out material properties in the vicinity of the resin flow front and the grid does not need to be adapted to the flow front location.

\section{Method}

In this section, the governing equations incl. the numerical discretisation of the resin flow through the fibre material are presented.

\subsection{Governing equations}

\subsubsection{Darcy's law}

Creeping flow of an incompressible Newtonian fluid through a porous medium is governed by Darcy's law [8]:

$$
\mathbf{u}=\mathbf{u}_{f}-\frac{1}{\mu \theta_{v}} \mathbf{K} \boldsymbol{\nabla} p, \quad \mathbf{q}=\theta_{v} \mathbf{u}
$$

Throughout the paper, bold, lower-case letters are vectors and bold, upper-case letters are matrices. $\nabla()$ is the gradient operator. In Eq. (1), $\mathbf{q}$ is the spatial Darcy flux (volumetric discharge), $\mathbf{u}$ is the velocity of the fluid, $\mathbf{u}_{f}$ is the bulk velocity of the porous medium, and $p$ is the pressure. The fluid, which will be referred to as the resin, is characterised by the dynamic viscosity, $\mu$, while the porous medium, which will be referred to as the fibre reinforcement, is characterised with the permeability tensor, $\mathbf{K}$, and the porosity, $\theta_{v}$. The permeability is depended on the porosity, fibre diameter, and the fibre architecture of the fibre material.

The pressure equation is derived by enforcing mass conservation, which means that the divergence of the volumetric discharge (Eq. (1)) must be in balance with any sources or sinks, $s$. Omitting $\theta_{v} \mathbf{u}_{f}$, the pressure equation becomes:

$$
\boldsymbol{\nabla} \cdot \mathbf{q}=s, \quad \boldsymbol{\nabla} \cdot\left(-\frac{1}{\mu} \mathbf{K} \boldsymbol{\nabla} p\right)=s
$$

As $\theta_{v} \mathbf{u}_{f}$ has been omitted, Eq. (2) only applies to cases where the bulk velocity of the fibre material is constant in all directions. Such cases include e.g. resin-injection pultrusion processes with a straight impregnation chamber, where the bulk velocity of the porous medium $\mathbf{u}_{f}$ is the profile-advancing pulling speed. This simplification also applies to cases where the fibre bed is stationary.

\subsubsection{The level-set method}

As the resin infiltrates the fibre reinforcement, the domain occupied by the resin expands. To track this development, the level-set method first introduced by Osher and Sethian [27] is used. In the level-set method, the interface location is represented implicitly by the zero-contour of a function, $\phi$, and each sub-domain, i.e. 
the domain occupied by the resin and the domain occupied by the dry fibre reinforcement, is represented by the sign of the function, $\phi$ :

$$
\left\{\begin{array}{lll}
\phi=0 & \text { on } & \Gamma_{\text {interface }} \\
\phi<0 & \text { in } & \Omega_{\text {resin }} \\
\phi>0 & \text { in } & \Omega_{\text {dry }}
\end{array}\right.
$$

The level-set function, $\phi$, is subject to advection from the velocity field determined in Eq. (1):

$$
\frac{\partial \phi}{\partial t}+\mathbf{u} \cdot \nabla \phi=0
$$

The level-set function is usually chosen to be a signed distance function due to its good properties for mass conservation and overall accuracy [16]. A signed distance function holds the absolute distance to the interface location, which is illustrated for a circular flow front in Fig. 1.

[Figure 1 around here]

As the level-set function is advected through time-integration of Eq. (4), it tends to lose its property of being a signed distance function. This property can be reestablished by solving

$$
\frac{\partial \phi}{\partial \tau}+\operatorname{sgn}(\phi)(\|\nabla \phi\|-1)=0
$$

to steady state, which is referred to as reinitialisation. While conventional level-set methods do not explicitly satisfy mass conservation near the flow front, it is noted that this type of reinitialisation ignores the initial interface location [31] and can lead to an additional discrepancy in mass conservation. Furthermore, continued reinitialisation of this type may severely move the interface location and, in addition, affect the contact angle towards boundaries [13]. This can be addressed by constrained, iterative schemes [17], or special single-step, non-iterative schemes [15].

\subsection{Numerical discretisation}

The discretisation is applied on a 2D Cartesian grid, with an equidistant spacing of $\Delta x$ and $\Delta y$. The FV grids are staggered, such that the pressure and level-set values share cell centres, while the spatial velocities, $\mathbf{u}$, lie on the cell faces. The FV schemes are derived for a cell, ()$_{i, j}$, with neighbouring cells to the west, east, south and north sides, denoted by ()$_{i-1, j},()_{i+1, j},()_{i, j-1}$, and ()$_{i, j+1}$, respectively. The face values of the cells ()$_{i, j}$, follow the same convention. For example, the east face of cell ()$_{i, j}$ is denoted as ()$_{i+1 / 2, j}$.

\subsubsection{Advection of the level-set}

The conservation law for the advection of the level-set equation (Eq. (1)) is approximated with an FV scheme by integrating over a control volume $[\Delta x \times \Delta y]$ :

$$
\int_{\Delta x \Delta y}\left(\frac{\partial \phi}{\partial t}+\mathbf{u} \cdot \nabla \phi\right) \mathrm{d} x \mathrm{~d} y=0
$$


Using Gauss' theorem, Eq. (6) for cell ()$_{i, j}$ is rewritten as:

$$
\frac{\partial \bar{\phi}_{i, j}}{\partial t}=\frac{1}{\Delta x}\left((u \hat{\phi})_{i+1 / 2, j}-(u \hat{\phi})_{i-1 / 2, j}\right)+\frac{1}{\Delta y}\left((v \hat{\phi})_{i, j+1 / 2}-(v \hat{\phi})_{i, j-1 / 2}\right)
$$

where $(\hat{)}$ indicates that the value is upwinded and $\bar{\phi}$ is the cell-average of $\phi$. For brevity, the $(\overline{)}$-notation will be dropped. The evaluation of face-values of the resin velocity, e.g. $u_{i+1 / 2, j}$, will be discussed in Sec. 2.2.3.

To evaluate the level-set function at cell faces, a high-order, upwinded 5th-order Weighted Essentially NonOscillatory (WENO5) interpolation scheme is applied. In this scheme, the face value of $\phi$ is evaluated based on a wide, five-cell stencil. The five-cell stencil consists of three, two-cell overlapping sub-stencils. Each sub-stencil is weighted in the full WENO5-stencil based on the local smoothness, meaning any sub-stencil that contain a jump in the level-set function is assigned a low weight. This property is important, as it allows the numerical framework to properly interpolate across any discontinuities in the level-set function. Consequently, the framework can remain stable when flow fronts merge or multiple flow fronts enter the WENO5 interpolation stencil. As research concerning WENO schemes is extensive and well-established, we refer to relevant literature for details about this scheme, e.g. Sec. 2.2 in Shu [35].

The WENO5 scheme is upwinded by favouring three cells to the upwind side. Formally, this means if $u>0$, $\hat{\phi}=\phi^{-}$, which favours three points towards the west side. Similarly, if $u<0, \hat{\phi}=\phi^{+}$is used, which favours three points towards the east side. If $u=0$, then $u \hat{\phi}=0$ no matter which direction the scheme is upwinded.

\subsubsection{Reinitialisation and the narrow-band approach}

The single step algorithm developed by Fu et al. [15] is used for reinitialisation of the level-set function. This algorithm is not iterative, it does not move the interface location (i.e. it does not affect local volume fractions), and the level-set function is not affected by continued reinitialisation. Furthermore, it is more accurate and significantly faster than comparable iterative schemes [15]. As the algorithm proposed in this paper deviates slightly from Fu et al. [15], the framework is briefly outlined below. The modifications mainly relate to how the interface is approximated at the cell-level and how ghost points around boundaries are treated (c.f. Sec. 2.2.4).

The algorithm consists of two successive steps that are conducted for every time increment.

1. The first step is to locate the cells that constitute the interface, which are to be kept fixed by excluding them from the reinitialisation step. See $\Omega_{f i x}$ in Fig. 2. These cells are identified by the following relations:

$$
\phi_{i, j} \phi_{i-1, j} \leq 0, \quad \text { or } \quad \phi_{i, j} \phi_{i+1, j} \leq 0, \quad \text { or } \quad \phi_{i, j} \phi_{i, j-1} \leq 0, \quad \text { or } \quad \phi_{i, j} \phi_{i, j+1} \leq 0
$$

Eq. (8) includes cells that are split by the interface ( $\phi$ changes sign over two neighbouring cells), and neighbouring cells where one or more cell centres coincide with the interface $(\phi=0)$.

The cells in $\Omega_{f i x}$ that are not completely filled, i.e. cells that are actually cut by the interface, enter $\Omega_{c u t}$ in addition to $\Omega_{f i x}$. All other cells in the level-set function are assigned large values, while maintaining the initial sign of the level-set, prior to the next step. 
with coefficients based on the normal to the level-set, $\mathbf{n}$, and the absolute distance to the interface, $\phi_{i, j}$ :

$$
a=-n_{x}, \quad b=-n_{y}, \quad c=n_{x} x+n_{y} y-\phi_{i, j}, \quad \mathbf{n}=\frac{\boldsymbol{\nabla} \phi}{\|\boldsymbol{\nabla} \phi\|}
$$

where the gradient of the level-set function can be estimated based on cells only in $\Omega_{f i x}$ using central differences when possible, and alternatively one-sided differences.

Once the interface in a cut-cell in $\Omega_{c u t}$ has been approximated by a line, the absolute distance to the interface is estimated in target cells, $\Omega_{t a r}$ in Fig. 2, which are the four vertical and horizontal neighbouring cells in both directions. The approximation of the absolute distance follows the relation [15]:

$$
\bar{d}=\beta d+(1-\beta) r
$$

where $d$ is the distance from the target cell to the approximated interface and $r$ is the distance from the target cell to the interface point. Both $d$ and $r$ can be calculated by simple geometrical operations. In Eq. (11), $\beta$ is a smooth Wendland function defined as [9]:

$$
\beta=(1-\chi)^{6}\left(1+6 \chi+\frac{35}{3} \chi\right), \quad \chi=\min \left(\frac{2 h}{3(\Delta x+\Delta y)}, 1\right)
$$

where $h$ can be determined based on geometrical operations (c.f. Fig. 2). From Eqs. (11-12) and Fig. 2 , it is noted that as $h$ approaches 0 , the estimate of the absolute distance to the interface improves. Furthermore, when $h=0$ and $\beta=1, d$ holds the true absolute distance to the interface, $\bar{d}=d$.

Throughout this step, cells in $\Omega_{t a r}$ are visited multiple times. As the best estimate of the absolute distance is the shortest estimated distance, the level-set function is updated by replacing $\phi$ with $\operatorname{sgn}(\phi) \cdot \min (|\phi|, \bar{d})$.

For further information about the reinitialisation scheme, please see Fu et al. [15].

[Figure 2 around here]

As the level-set function is only defined locally in a narrow band of the four neighbouring cells to $\Omega_{c u t}$, there is no need to advect $\phi$ in the full domain. In fact, as just cells in $\Omega_{f i x}$ are needed to reestablish the level-set function, only cells adjacent to $\Omega_{f i x}$ are advected in Eq. (7). This greatly reduces the computation time, as only a fraction of the domain needs to be advected.

\subsubsection{Pressure equation and face velocities}

The FV scheme of the pressure equation, Eq. (2), is established by integrating over a control volume $[\Delta x \times$ $\Delta y]$ and applying Gauss' theorem:

$$
\frac{1}{\Delta x}\left(\left(\frac{K_{1}}{\mu} \frac{\partial p}{\partial x}\right)_{i+1 / 2, j}-\left(\frac{K_{1}}{\mu} \frac{\partial p}{\partial x}\right)_{i-1 / 2, j}\right)+\frac{1}{\Delta y}\left(\left(\frac{K_{2}}{\mu} \frac{\partial p}{\partial y}\right)_{i, j+1 / 2}-\left(\frac{K_{2}}{\mu} \frac{\partial p}{\partial y}\right)_{i, j-1 / 2}\right)=s_{i, j}
$$


which is a conventional FV scheme of a Poisson's equation for anisotropic diffusion. Eq. (13) is valid for orthotropic materials with principal directions following the $x$ - and $y$-axes. This implies that there are no offdiagonal terms in the permeability tensor. The scheme can easily be expanded to general anisotropic materials, see e.g. Sec. 2.1 in van Es et al. [14].

In Eq. (13), the pressure gradient at the cell faces is evaluated by central differences and the face value of local material properties are determined by harmonic means (see e.g. van Es et al. [14]). Viscosity varies from cell to cell based on whether cells are occupied by resin or air:

$$
\mu_{i, j}=\mu_{a i r}\left(1-\alpha_{i, j}\right)+\left(\mu_{r e s}-\mu_{a i r}\right) \alpha_{i, j}
$$

where $\alpha_{i, j}=[0 ; 1]$ is the volume fraction of the resin. The viscosity of air is much lower than the one of resin, whereby it is assigned a low value $\mu_{\text {air }}=10^{-9} \mu_{\text {res }}$.

Finally, the face-velocities of $\mathbf{u}$ are determined by Eq. (1). When there are no off-diagonal terms in the permeability tensor, the velocity at e.g. the east face becomes:

$$
u_{i+1 / 2, j}=\left(\frac{K_{1}}{\mu \theta_{v}}\right)_{i+1 / 2, j} \frac{p_{i+1, j}-p_{i, j}}{\Delta x}+\left(u_{f}\right)_{i+1 / 2, j}
$$

It is noted that the scheme does not smear out (smooth) the material properties over multiple cells near the interface, as only material properties in cut-cells, $\Omega_{c u t}$, depend on the level-set. Furthermore, if volume fractions are based on data available within the band of fixed cells, $\Omega_{f i x}$, then the pressure and the associated resin velocity will not be affected by reinitialisation of the level-set function. In this paper, the volume fraction is calculated by geometrical operations based on the approximated interface in Eq. (9), which means that the volume fractions are indeed unaffected by reinitialisation.

As an alternative to harmonic means, it is possible to evaluate material coefficients at the cell faces by using the location of the interface relative to two split cells according to Liu et al. [21]. However, we did not see an improvement in the order or magnitude of accuracy using that approach.

\subsubsection{Boundary conditions}

The boundary conditions in LCM are in- and outlets, together with the locations of mould walls. The boundary conditions are applied to the FV scheme of the pressure equation (Eq. (13)), and the advection (Eq. (7)) and reinitialisation of the level-set function (Sec. 2.2.2).

For the pressure equation, in- or outflow boundary conditions are enforced via a prescribed pressure (Dirichlet boundary type) or an inflow rate of resin via the pressure gradient (Neumann boundary type). At mould walls, the pressure gradient normal to the mould side is set to zero, which implies that no resin flows through this boundary. This condition also applies to symmetry lines.

For the level-set function, two ghost points are included around the boundary, which is sufficient if the wide WENO5 stencils are upwinded away from the boundaries. For symmetry or periodic boundary conditions, the 
ghost points are filled with copies from appropriate cells near the boundaries. For other boundary conditions, that is inlets, outlets and mould walls, the ghost points are filled with first-order, linear extrapolations. This method essentially extrapolates the contact line between the resin front and the boundary to the ghost points. After appropriate values have been filled into ghost-points, reinitialisation is applied. It is noted that this approach is not stable for conventional, iterative reinitialisation schemes [10], but we saw no stability issues when using the single-step reinitialisation scheme by Fu et al. [15].

\subsubsection{Solution algorithm and time-stepping scheme}

The solution algorithm that we propose consists of solving the pressure equation (Eq. (13)) implicitly and subsequently advecting the level-set function (Eq. (7)) explicitly. Defining $L(\phi)=\partial \phi / \partial t$, and using a temporal discretisation of $2^{\text {nd }}$-order of the predictor-corrector type, the solution algorithm is:

1. At time step $n$, build a system of equations and solve implicitly for pressure, $p^{n}$, (Eq. (13)) based on the interface location at time $n$. Calculate cell-face velocities of cells in and adjacent to $\Omega_{f i x}$.

2. Build $L\left(\phi^{n}\right)$ according to Eq. (7) and advect the level-set function:

$$
\tilde{\phi}^{(n+1)}=\phi^{n}+\Delta t \cdot L\left(\phi^{n}\right)
$$

3. Repeat step 1 for $\tilde{\phi}^{n+1}$ and build $L\left(\tilde{\phi}^{n+1}\right)$ to correct:

$$
\phi^{n+1}=\phi^{n}+\frac{\Delta t}{2}\left(L\left(\tilde{\phi}^{n+1}\right)+L\left(\phi^{n}\right)\right)
$$

4. Update ghost points for $\phi^{n+1}$ according to Sec. 2.2.4.

5. Reinitialise $\phi^{n+1}$ according to Sec. 2.2.2.

6. Step forward in time, $t \rightarrow t+\Delta t, n \rightarrow n+1$, and repeat steps 1-5 while $t<t_{\text {end }}$.

The time step is constrained by $\Delta t=\mathrm{CFL} \cdot \min \left(\frac{\Delta x, \Delta y}{|\mathbf{u}|}\right)$, with a conservative choice for stability being $\mathrm{CFL} \approx 0.5[26]$.

\subsubsection{Optional modifications}

Based on the numerical experiments in Sec. 3, the scheme outlined in Secs. 2-2.2.5 performs well in the reported cases related to LCM. However, we suggest two possible modifications that can be applied if global volume conversation is important for a particular problem, or if the level-set function becomes flat or steep near the interface.

Please note that if not explicitly otherwise stated, the modifications are not applied in this paper.

Volume correction step. As stated in Sec. 2.1.2, level-set methods do generally not explicitly satisfy volume conservation near the flow front. To maintain global conservation, an optional correction step is proposed:

$$
\sum_{i}^{N_{x}} \sum_{j}^{N_{y}}\left(\alpha_{i, j}^{n+1}-\alpha_{i, j}^{n}\right) \Delta x \Delta y=\Delta t^{*} \sum_{i}^{N_{s}} s_{i}
$$


The first term in Eq. (18) corresponds to the calculated change in the infused volume from time step $n$ to $n+1$ and the latter is the net in- or outflow through sources/sinks (in- and outlets). Please note that the latter term must be accumulated over any sub-steps for non-constant flow rates. The asterisk indicates that $\Delta t^{*}$ is the time duration for which Eq. (18) is in balance. The correction step consists of calculating $\Delta t^{*}$ from Eq. (18) after a successive time step, and then adjust how much forward in time the algorithm advances from $n$ to $n+1$. This is done by replacing $\Delta t$ with $\Delta t^{*}$ in step 6, Sec. 2.2.5. The correction step is demonstrated in Sec. 3.1.3.

Steep or flat interface. In certain situations, the level-set function may become steep or flat in the vicinity of the interface as it is advected in the velocity field. This means that the level-set loses its property of being a signed distance function at this location. Based on the numerical validations in Section 3, this has generally not shown to be an issue for LCM simulation. However, for cases where the bulk velocity of the fibres is significant, this can take place and introduce oscillations into the solution. To overcome this problem, the renormalisation step proposed by Fu et al. [15] is applied. The step consists of recovering the signed distance function property of the level-set function before reinitialisation of cells in $\Omega_{t a r}$. This is done by replacing $\phi$ with $\phi /\|\nabla \phi\|$ in $\Omega_{f i x}$. The renormalisation step is demonstrated in Sec. 3.4.

\subsubsection{Possible extension to $3 D$}

As it was shown in this section, the flow front location is represented by the zero-contour of a signed distance function. If the framework were to be extended into $3 \mathrm{D}$, the flow front location would be represented by the zero iso-surface of a signed distance function. This means the approximation of the interface would be a surface in Eqs. (9-10) [15].

For the calculation of the pressure field, a 3D implementation would need to consider the fluxes in the $z$ direction in Eq. (13). This also applies to the subsequent advection of the level-set function, where the velocity field in the $z$-direction will enter in Eq. (7).

\section{Numerical validations and discussion}

The numerical framework is validated against a variety of situations that occur in LCM processes. The cases include radial injection, edge-effects in linear injection tests (often referred to as race-tracking), merging of the resin fronts, and resin-injection pultrusion. All cases are compared to analytical solutions, experiments, and/or numerical simulations. An overview of the different cases can be seen in Fig. 3. Relevant parameters and material properties are listed in Table 1.

The presented numerical results are based on an in-house code written in the technical programming language MATLAB ${ }^{\mathrm{TM}}$.

[Figure 3 around here] 


\subsection{Radial injection, incl. rate of convergence}

The first test demonstrates the convergence behaviour of the numerical framework and verifies that anisotropy is correctly handled. Finally, the volume correction method is demonstrated. The case is a radial injection test, where resin is injected at a constant flow rate, $s$, at the centre of a fibre bed. The case is illustrated in Fig. 3(a) and material data can be found in Table 1.

To derive an analytical solution for this case, a coordinate transformation is conducted [40]:

$$
x_{f}^{\prime}=\sqrt{\frac{K^{\prime}}{K_{1}}} x_{f}, \quad y_{f}^{\prime}=\sqrt{\frac{K^{\prime}}{K_{2}}} y_{f}
$$

where $K^{\prime}$ is the quasi-isotropic permeability, $K^{\prime}=\sqrt{K_{1} K_{2}}$. In the quasi-isotropic plane, the flow front location is the same in all directions for a radial injection test, $x_{f}^{\prime}=y_{f}^{\prime}=r_{f}^{\prime}$, whereby the total volume of the resin in the mould, $S$, and the flow front location, $r_{f}^{\prime}$, is found by the relation:

$$
S=\int_{0}^{t} s \mathrm{~d} t+S_{0}=s \cdot t+S_{0}, \quad r_{f}^{\prime}=\sqrt{\frac{S}{\pi}}
$$

\subsubsection{Convergence behaviour (isotropic fibre bed)}

The convergence behaviour of the numerical framework is evaluated by starting a simulation with a circular resin domain of $r_{0 f}$, for which the level-set function can be evaluated analytically:

$$
\phi_{0}^{e}=\sqrt{(x-0.5)^{2}+(y-0.5)^{2}}-r_{0 f}
$$

The radius, $r_{0 f}$, is chosen such that $S_{0}=0.2 \mathrm{~m}^{2}$. Over 200 time increments with constant time stepping, the initial resin content is doubled at a rate of $s / w_{z}=1 \mathrm{~m}^{2} / \mathrm{s}$ (normalised w.r.t. the thickness of the fibre bed). The $L_{2}$ and $L_{\infty}$-norm of the error in the interface location, together with the discrepancy between the actual and expected area, $S_{\text {error }}$, are evaluated as in Fu et al. [15]:

$$
\phi_{\text {error }, L_{2}}=\sqrt{\frac{1}{N} \sum_{i}^{N} \sum_{j}^{N} H_{i, j}\left(\phi_{i, j}-\phi_{i, j}^{e}\right)^{2}}, \quad \phi_{\text {error }, L_{\infty}}=\max _{i, j}\left|\phi_{i, j}-\phi_{i, j}^{e}\right|, \quad S_{\text {error }}=\left|\frac{S-S^{e}}{S^{e}}\right|
$$

where $H_{i, j}$ is a window function that only includes cells in the narrow band around the interface, and $N$ is the number of cells in the narrow band. The area of the resin domain, $S$, is calculated using simplices [12] that is $2^{\text {nd }}$-order accurate.

As it can be seen in Fig. 4, the numerical framework maintains between $1^{\text {st }}$ and $2^{\text {nd }}$-order accuracy for the instances tested. Furthermore, it is noted that for a relatively coarse grid of $31 \times 31$, the infused volume is conserved with an error less than $1 \%$, and the flow front location is estimated with an error of less than $0.01 \mathrm{~m}$. [Figure 4 around here] 


\subsubsection{Convergence behaviour, cont., incl. computation time (anisotropic fibre bed)}

For fibre beds with varying degree of anisotropy, $\psi=K_{1} / K_{2}$, the flow front position is now estimated based on Eqs. (19-20). The level-set is initialised such that the resin occupies a circular domain with a radius of $r_{0 f}=0.04 \mathrm{~m}$ at the beginning of the simulation. A total of $S / w_{z}=0.15 \mathrm{~m}^{2}$ is injected (normalised w.r.t. the thickness of the fibre bed) at a constant rate of $s / w_{z}=1 \mathrm{~m}^{2} / \mathrm{s}$. All simulations are conducted with time-steps based on the CFL criteria, c.f. Sec. 2.2.5, with $C F L=0.25,0.5$ and 0.75 .

As it can be seen in Fig. 5, when comparing to the analytical solution, the numerical solution appears to be ahead. This corresponds to the expected behaviour of a forward-based, explicit scheme. The solution improves with decreasing time step size for all degrees of anisotropy tested and no apparent oscillations were observed in any of the solutions.

For this particular example, each full simulation takes on average 7 seconds of computation on a single CPU (laptop, $2.8 \mathrm{GHz}$, Core i7, 7700HQ). Of the 7 seconds, approximately 1.5 seconds ( $\approx 20 \%$ ) is spent on operations related to the movement of the flow front. This includes advection and reinitialisation of the level-set, calculation of volume fractions, and updating ghost points.

[Figure 5 around here]

\subsubsection{Volume correction step}

For the case presented in Sec. 3.1.1, the accumulation in the error of the infused volume is shown in Fig. 6. The error is shown with and without the application of the volume correction step presented in Sec. 2.2.6. The volumes used for determining the errors are calculated using simplices [12]. As it can be seen in Fig. 6, the error in conservation is small $(<0.4 \%)$, but with the volume correction scheme the infused volume does not continue to grow. It is noted that while the correction step ensures that the error in global volume conservation does not accumulate, it does not restore mass conversation on a cell-level basis near the interface.

[Figure 6 around here]

\subsection{Race-tracking (edge-effects)}

Race-tracking is an edge-effect that can occur in LCM. Race-tracking can be the desired effect that takes place if layers of flow media (also called resin bleeders) are included in the fibre layup. As such layers are normally highly permeable, they aid feeding in resin to the composite part and are an important tool in the optimisation of the resin impregnation process. Race-tracking can also be an unwanted effect that can occur in small gaps between sandwich panels or near mould sides/walls and the fibre material. Either way, it represents an important feature in the simulation of LCM, and it demonstrates that local jumps in material properties are handled correctly.

In the numerical framework, the race-track is treated as an area with increased permeability. The permeabil- 
ity is based on the pressure drop for viscous flow in a rectangular channel, given in Young [42]:

$$
K_{r t}=\frac{w_{z c}^{2}}{96}\left(1-\frac{192 w_{z c}}{\pi^{5} w_{x c}} \tanh \left(\frac{\pi w_{x c}}{2 w_{z c}}\right)\right)
$$

The test case is illustrated in Fig. 3(b), where dimensions $w_{x c}$ and $w_{z c}$ are identified. The material properties are listed in Table 1.

In this test case, the resin is injected with a constant pressure from the west side of a solid mould. The east side of the mould is open, whereby atmospheric pressure is applied. The race-track is located towards the north mould wall. The numerical solution is compared to Yang et al. [41], who revisits the experiments by Young [42]. In absence of the race-track (a homogeneous fibre bed), the flow front follows the relation:

$$
t=\frac{\mu_{R} \theta_{v}}{2 K_{1} \Delta p} x_{f}^{2}
$$

The results are illustrated in Fig. 7.

Based on the reported results in $[42,41]$, the numerical scheme correctly captures how the resin advances faster in the race-track. Furthermore, in absence of the race-track, the error between the analytical solution and numerical scheme is below $0.2 \%$ for the three time-instances illustrated.

[Figure 7 around here]

\subsection{Radial injection with flow front merge}

When the resin is infused through multiple injection points, the resin fronts will merge at some point during the impregnation step. To demonstrate how the numerical framework handles this flow characteristic, the experiments presented in Shimada et al. [33] are the basis for this validation. As it is illustrated in Fig. 3(c), the resin is injected through two inlets with radius, $r_{0}$. When the two resin fronts merge, a weld line between the fronts develops.

From the reported flow front positions in the warp and weft-directions by Shimada et al. [33] (c.f. Fig. 9), the permeabilities are estimated based on the inlet-scaling method described in Weitzenböck et al. [40]. Measurements between 40 and $90 \mathrm{~s}$ are used, which means that only measurements before the formation of the weld line are used. The estimated permeabilities and other relevant material data are listed in Table 1. Using the estimated permeabilities, the flow front positions before and throughout the formation of the weld line can now be simulated. This is shown for various time instances in Fig. 8.

The flow front predictions follow the reported experiments well as seen in Fig. 9, with the exception that the initial formation of the weld line appears to be delayed. For this kind of free-surface method, a numerical artefact of this type is to be expected. The reason for this is when the distance between the two flow fronts is shorter than one cell-length, velocities from the opposing resin domain will enter the interpolation stencils. This will artificially slow down the advection of the level-set function and cause a stall in the formation of the weld line. It is noted that the numerical results reported by Shimada et al. [33] demonstrated similar behaviour. [Figures 8 and 9 around here] 


\subsection{Resin-injection pultrusion (RIP)}

RIP is a process used to manufacture composite profiles with a constant cross-section. In RIP, the dry fibre material is pulled through a die, where the material is impregnated with a resin by means of resin-injection.

For the simulation of the impregnation step in a RIP process, the profile-advancing pulling speed enters Eq. (1) as the bulk velocity of the fibre material, $\mathbf{u}_{f}$. The test case concerns pultrusion of a thin bar where the resin is injected through a point at the centreline with a constant flow rate. The case is illustrated in Fig. 3(d) and material properties together with boundary conditions are listed in Table 1. The results are compared to Liu [22] in Fig. 10, where it can be seen that an acceptable agreement between the two numerical solutions is obtained.

In this particular example, the level-set function tends to become steep near the interface, specifically in the backflow of the inlet. As the nature of the problem is a stationary process, the interface will quickly slow down and eventually come to a stop. However, the resin will still flow from the inlet and push the edge of the level-set function backwards, which will cause the level-set to become steep. To overcome this issue, the level-set is renormalised before reinitialisation by applying the correction step (c.f. Sec. 2.2.6) for every tenth time increment. The effect of the renormalisation step is illustrated in Fig. 11, where it can be seen that the renormalisation step corrects the level-set function without moving the interface location.

[Figure 10 around here]

[Figure 11 around here]

\section{Conclusion}

In this paper, a numerical framework for the simulation of liquid composite moulding using a FV scheme and the level-set method was presented. The framework consists of two successive steps. First, the pressure is solved implicitly, and subsequently, the resin flow front is advected explicitly. The numerical framework was verified against cases that represent a variety of situations that occur in LCM processes. These cases included analytical solutions, numerical simulations, and experiments of radial injection tests, edge-effects (race-tracking), merging of resin flow fronts, and resin-injection pultrusion. We highlight the following findings and conclusions for using level-sets in liquid composite moulding:

- High-order WENO stencils and a special, single step reinitialisation scheme allow the resin flow front to be advected with CFL-sized time steps without introducing any artificial smoothing and without overfilling cells;

- The numerical framework achieved convergence with a spatial accuracy between $1^{\text {st }}$ and $2^{\text {nd }}$-order;

- Conservation of volume is acceptable ( $<1 \%$ for the reported examples), and global conservation can be ensured by a simple correction step;

- The operations related to the movement of the resin flow front only take around $20 \%$ of the total CPU-time in the case reported. 


\section{Acknowledgements}

This work is funded by the Danish Council for Independent Research - Technology and Production Sciences (Grant no. DFF-6111-00112: Modelling the multi-physics in resin injection pultrusion (RIP) of complex industrial profiles).

\section{References}

[1] Acheson, J.A., Simacek, P., Advani, S.G., 2004. The implications of fiber compaction and saturation on fully coupled VARTM simulation. Composites Part A: Applied Science and Manufacturing 35, 159-169.

[2] Bickerton, S., Abdullah, M.Z., 2003. Modeling and evaluation of the filling stage of injection/compression moulding. Composites Science and Technology 63, 1359-1375.

[3] Bickerton, S., Advani, S.G., 1999. Characterization and modeling of race-tracking in liquidcomposite molding processes. Composites Science and Technology 59, 2215-2229.

[4] Binétruy, C., Hilaire, B., Pabiot, J., 1997. The interactions between flows occurring inside and outside fabric tows during rtm. Composites Science and Technology 57, 587-596.

[5] Bruschke, M.V., Advani, S.G., 1990. A finite element/control volume approach to mold filling in anisotropic porous media. Polymer Composites 11, 398-405.

[6] Bruschke, M.V., Advani, S.G., 1994. A numerical approach to model non-isothermal viscous flow through fibrous media with free surfaces. International Journal for Numerical Methods in Fluids 19, 575-603.

[7] Comminal, R., Spangenberg, J., Hattel, J.H., 2015. Cellwise conservative unsplit advection for the volume of fluid method. Journal of Computational Physics 283, $582-608$.

[8] Darcy, H., 1856. Les fontaines publiques de la ville de Dijon. Victor Dalmont, Libraire des Corps imperiaux des ponts et chaussées et des mines .

[9] Dehnen, W., Aly, H., 2012. Improving convergence in smoothed particle hydrodynamics simulations without pairing instability. Monthly Notices of the Royal Astronomical Society 425, 1068-1082.

[10] Della Rocca, G., Blanquart, G., 2014. Level set reinitialization at a contact line. Journal of Computational Physics 265, 34-49.

[11] DeParseval, Y., Pillai, K.M., Advani, S.G., 1997. A simple model for the variation of permeability due to partial saturation in dual scale porous media. Transport in Porous Media 27, 243-264.

[12] Detrixhe, M., Aslam, T.D., 2015. From level set to volume of fluid and back again at second-order accuracy. International Journal for Numerical Methods in Fluids 80, 231-255.

[13] Ervik, Å., 2016. Comment on the level-set method used in 'Numerical study on mobilization of oil slugs in capillary model with level set approach'. Engineering Applications of Computational Fluid Mechanics 10, 466-472. 
[14] van Es, B., Koren, B., de Blank, H.J., 2016. Finite-volume scheme for anisotropic diffusion. Journal of Computational Physics 306, 422-442.

[15] Fu, L., Hu, X.Y., Adams, N.A., 2017. Single-step reinitialization and extending algorithms for level-set based multi-phase flow simulations. Computer Physics Communications 221, 63-80.

[16] Gibou, F., Fedkiw, R., Osher, S., 2018. A review of level-set methods and some recent applications. Journal of Computational Physics 353, 82-109.

[17] Hartmann, D., Meinke, M., Schröder, W., 2010. The constrained reinitialization equation for level set methods. Journal of Computational Physics 229, 1514-1535.

[18] Hirt, C., Nichols, B., 1981. Volume of fluid (VOF) method for the dynamics of free boundaries. Journal of Computational Physics 39, 201-225.

[19] Jung, Y., Kim, S.J., Han, W.S., 2013. Numerical simulation of RTM process using the extended finite element method combined with the level set method. Journal of Reinforced Plastics and Composites 32, 308-317.

[20] Liu, B., Bickerton, S., Advani, S.G., 1996. Modelling and simulation of resin transfer moulding (RTM) - Gate control, venting and dry spot prediction. Composites Part A: Applied Science and Manufacturing $27,135-141$.

[21] Liu, X.D., Fedkiw, R.P., Kang, M., 2000. A Boundary Condition Capturing Method for Poisson's Equation on Irregular Domains. Journal of Computational Physics 160, 151-178.

[22] Liu, X.L., 2003. A finite element/nodal volume technique for flow simulation of injection pultrusion. Composites Part A: Applied Science and Manufacturing 34, 649-661.

[23] Michaud, V., 2016. A Review of Non-saturated Resin Flow in Liquid Composite Moulding processes. Transport in Porous Media 115, 581-601.

[24] Mohan, R.V., Ngo, N.D., Tamma, K.K., 1999. On a pure finite-element-based methodology for resin transfer mold filling simulations. Polymer Engineering and Science 39, 26-43.

[25] Nordlund, M., Michaud, V., 2012. Dynamic saturation curve measurement for resin flow in glass fibre reinforcement. Composites Part A: Applied Science and Manufacturing 43, 333-343.

[26] Osher, S., Fedkiw, R., 2006. Level set methods and dynamic implicit surfaces. volume 153. Springer Science \& Business Media.

[27] Osher, S., Sethian, J.A., 1988. Fronts propagating with curvature-dependent speed: Algorithms based on Hamilton-Jacobi formulations. Journal of Computational Physics 79, 12-49.

[28] Patiño-Arcila, I.D., Vanegas-Jaramillo, J.D., 2018. Modeling and simulation of filling in dual-scale fibrous reinforcements: state of the art and new methodology to quantify the sink effect. Journal of Composite Materials 52, 1915-1946.

[29] Pillai, K.M., 2004. Modeling the Unsaturated Flow in Liquid Composite Molding Processes: A Review and Some Thoughts. Journal of Composite Materials 38, 2097-2118. 
[30] Roy, T., Dulmes, C., Pillai, K.M., 2005. Experimental Investigations of the unsaturated flow in Liquid Composite Molding, in: Proceedings of the 5th Canadian International Composites Conference, pp. 1-12.

[31] Russo, G., Smereka, P., 2000. A Remark on Computing Distance Functions. Journal of Computational Physics 163, 51-67.

[32] Sandberg, M., Hattel, J.H., Spangenberg, J., 2018. Numerical modelling and optimisation of fibre wet-out in resin-injection pultrusion processes, in: European Conference on Composite Materials, Athens, Greece.

[33] Shimada, Y., Matsuzaki, R., Takahashi, A., 2016. Numerical simulation of molding-defect formation during resin transfer molding. Advanced Composite Materials 25, 17-32.

[34] Shojaei, A., Ghaffarian, S.R., Karimian, S.M., 2003. Modeling and Simulation Approaches in the Resin Transfer Molding Process: A Review. Polymer Composites 24, 525-544.

[35] Shu, C.W., 2009. High Order Weighted Essentially Nonoscillatory Schemes for Convection Dominated Problems. SIAM Review 51, 82-126.

[36] Šimáček, P., Advani, S.G., 2004. Desirable features in mold filling simulations for liquid composite molding processes. Polymer Composites 25, 355-367.

[37] Soukane, S., Trochu, F., 2006. Application of the level set method to the simulation of resin transfer molding. Composites Science and Technology 66, 1067-1080.

[38] Trochu, F., Gauvin, R., Gao, D.M., 1993. Numerical analysis of the resin transfer molding process by the finite element method. Advances in Polymer Technology 12, 329-342.

[39] Trochu, F., Ruiz, E., Achim, V., Soukane, S., 2006. Advanced numerical simulation of liquid composite molding for process analysis and optimization. Composites Part A: Applied Science and Manufacturing 37, 890-902.

[40] Weitzenböck, J.R., Shenoi, R.A., Wilson, P.A., 1999. Radial flow permeability measurement. Part A: Theory. Composites Part A: Applied Science and Manufacturing 30, 781-796.

[41] Yang, J., Jia, Y., Ding, Y., He, H., Shi, T., An, L., 2010. Edge effect in RTM processes under constant pressure injection conditions. Journal of Applied Polymer Science 118, 1014-1019.

[42] Young, W.b., 1997. Analysis of the edge effect in resin transfer molding. Composites Part A: Applied Science and Manufacturing 28A, 817-822. 


\section{List of Figures}

1 Illustration of a signed distance function specifying the interface location by the signed, absolute distance to the interface, and how the zero-contour splits the domain. . . . . . . . . . . . . . . 19

2 Illustration of how the absolute distance to the interface is approximated by the single step reinitialisation scheme by Fu et al. [15]. . . . . . . . . . . . . . . . . . . . . . . . 19

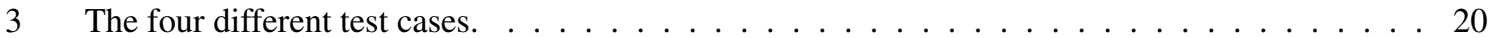

4 Referring to Fig. 3(a), convergence behaviour of the isotropic radial injection test. . . . . . . . 20

5 Referring to Fig. 3(a), this figure illustrates the flow front positions after a volume of $S / w_{z}=$ $0.2\left[\mathrm{~m}^{2}\right]$ has been injected. The flow fronts are shown for different degrees of anisotropy, $\psi=K_{1} / K_{2}=(10,5,2.5,1,1 / 2.5,1 / 5,1 / 10)$ and is compared to the analytical solution (ref.) for different time step sizes, $\Delta t$. The filled contour illustrates the resin domain for the isotropic

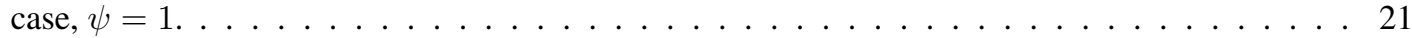

6 The error of the infused volume for the example in Sec. 3.1.1 with and without the application of the volume correction scheme. . . . . . . . . . . . . . . . . . . . . . . . . . . . . . 21

7 Referring to Fig. 3(b), (a) illustrates the flow front after $t=10,28.4$ and $60 \mathrm{~s} . K_{1 c}=K_{1}$ is for a homogeneous fabric, where the flow front location can be compared to an analytical solution. $K_{1 c}=K_{r t}$ is when a highly permeable race-track near the mould edge is present. For this case, the solution is compared to Yang et al. [41], who revisits the experiments by Young [42]. (b)

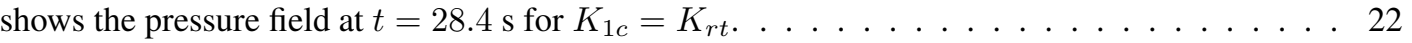

8 Referring to Fig. 3(c), the figure illustrates flow front positions in increments of $20 \mathrm{~s}$. The filled contour is the resin domain after $160 \mathrm{~s} \ldots \ldots \ldots \ldots \ldots \ldots$

9 Referring to Fig. 3(c), the figure illustrates the development of the flow front in the warp and weft direction, together with the weld line. (ref.) are experimental results from Shimada et al. [33] 23

10 Resin-injection pultrusion (Fig. 3(d)). The flow front is shown for time-increments of $10 \mathrm{~s}$ and is compared to numerical results from Liu [22] (ref.). The filled contour corresponds to the resin

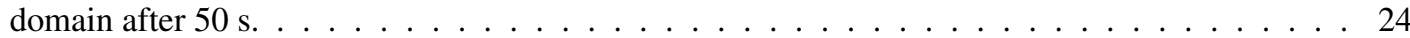

11 The level-set function at the centre line for resin-injection pultrusion at steady state with and without the application of renormalisation (cf. Sec. 2.2.6). While the interface location is the same (the intersection with the $x$-axis), the level-set function becomes steep in the vicinity of the interface when the renormalisation is not applied for this particular example. . . . . . . . . . 24 


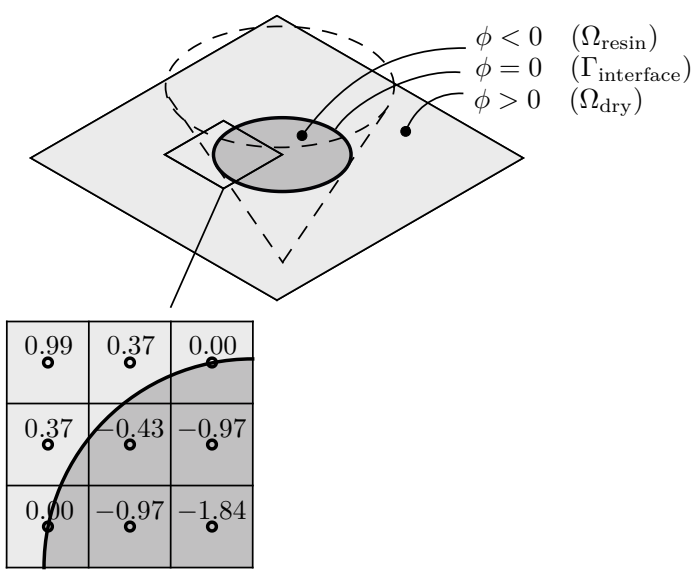

Figure 1: Illustration of a signed distance function specifying the interface location by the signed, absolute distance to the interface, and how the zero-contour splits the domain.

Interface point

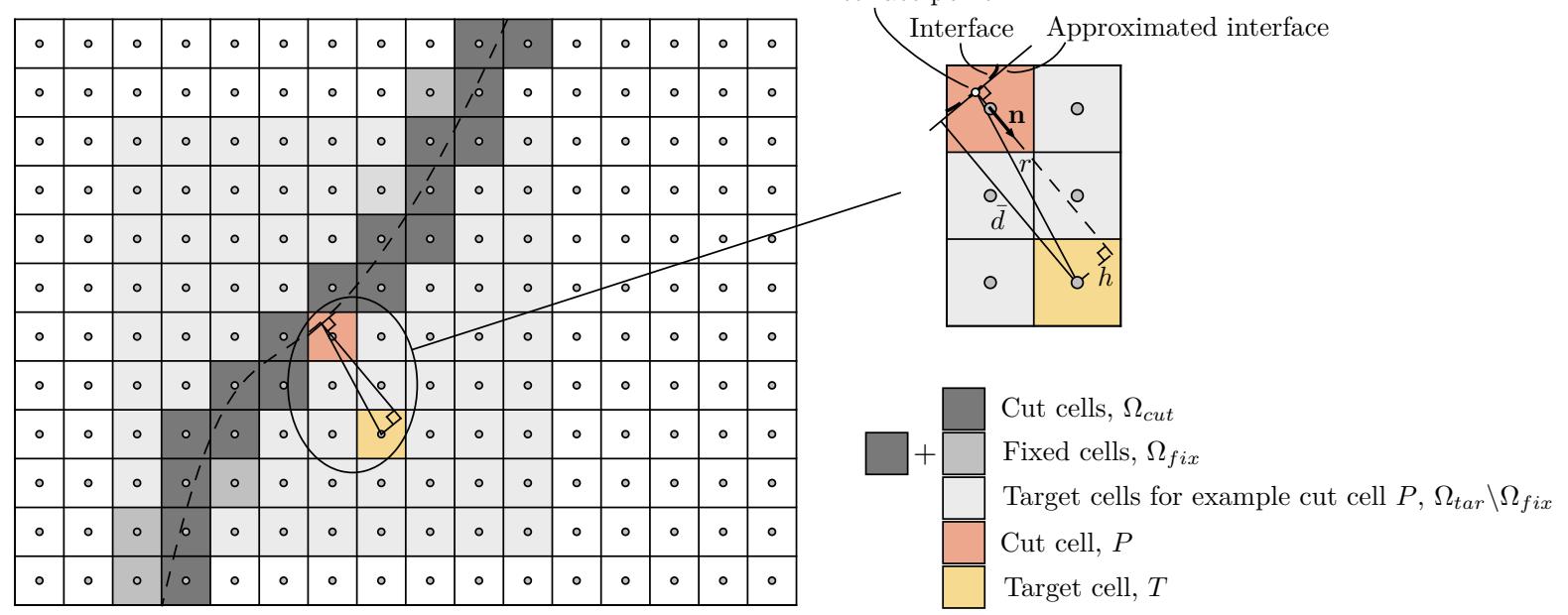

Figure 2: Illustration of how the absolute distance to the interface is approximated by the single step reinitialisation scheme by Fu et al. [15]. 
(a) Radial injection

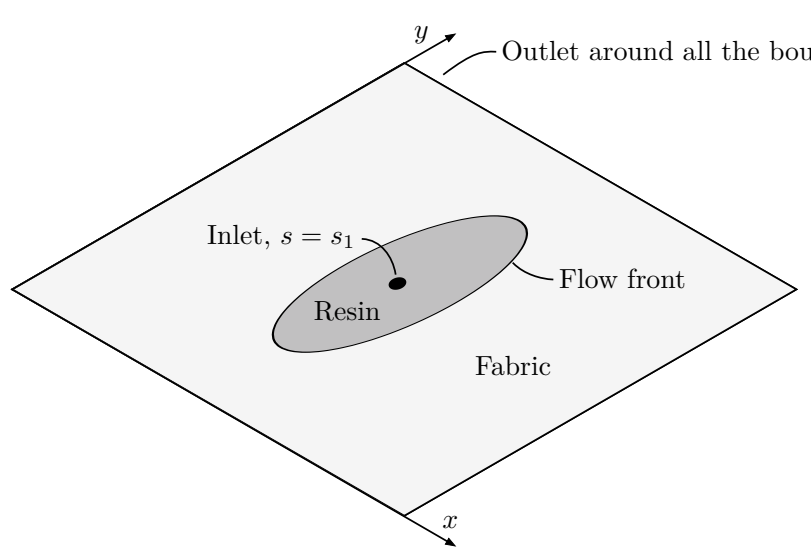

(c) Flow-front merge

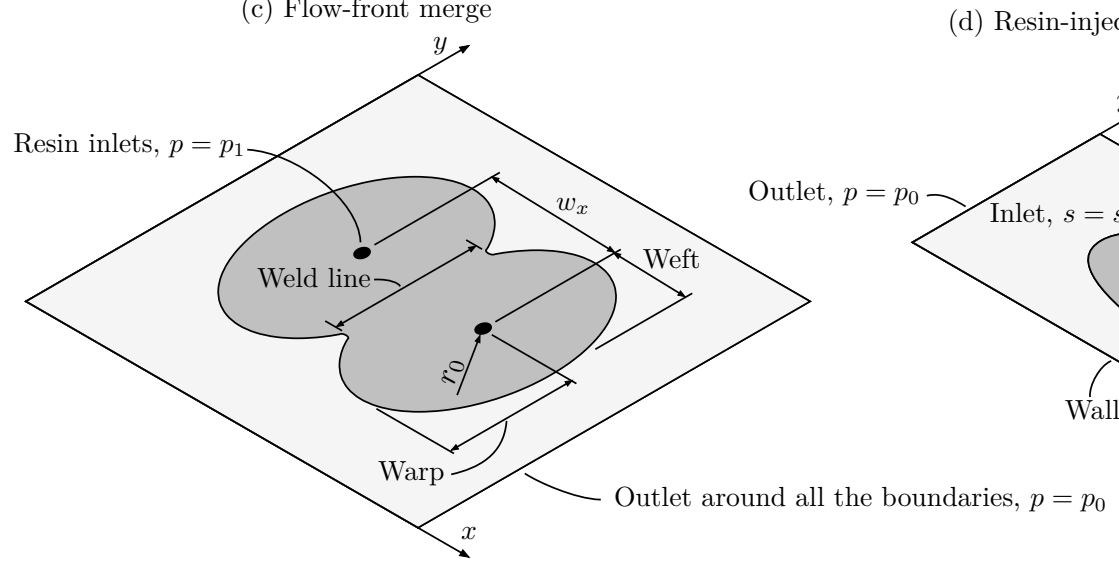

(d) Resin-injection pultrusion

Figure 3: The four different test cases.

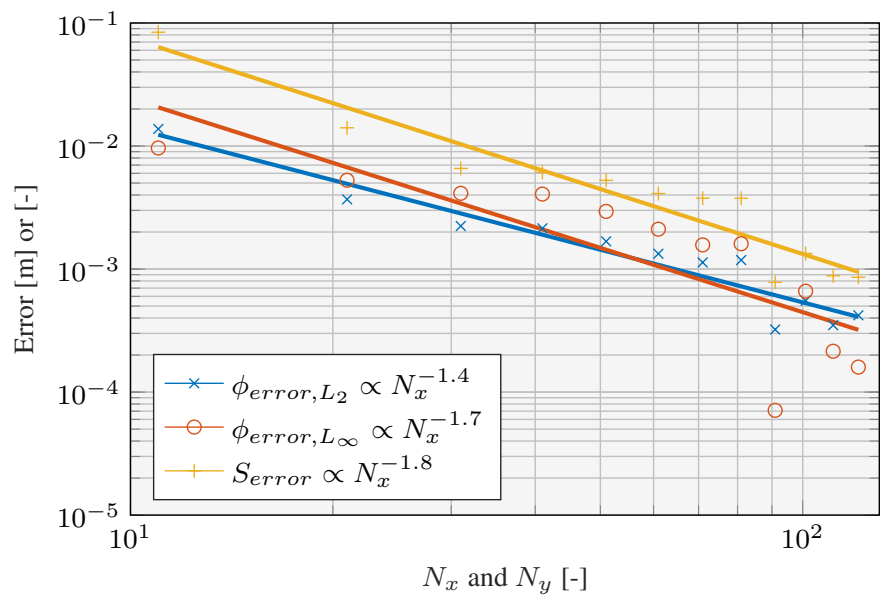

Figure 4: Referring to Fig. 3(a), convergence behaviour of the isotropic radial injection test. 


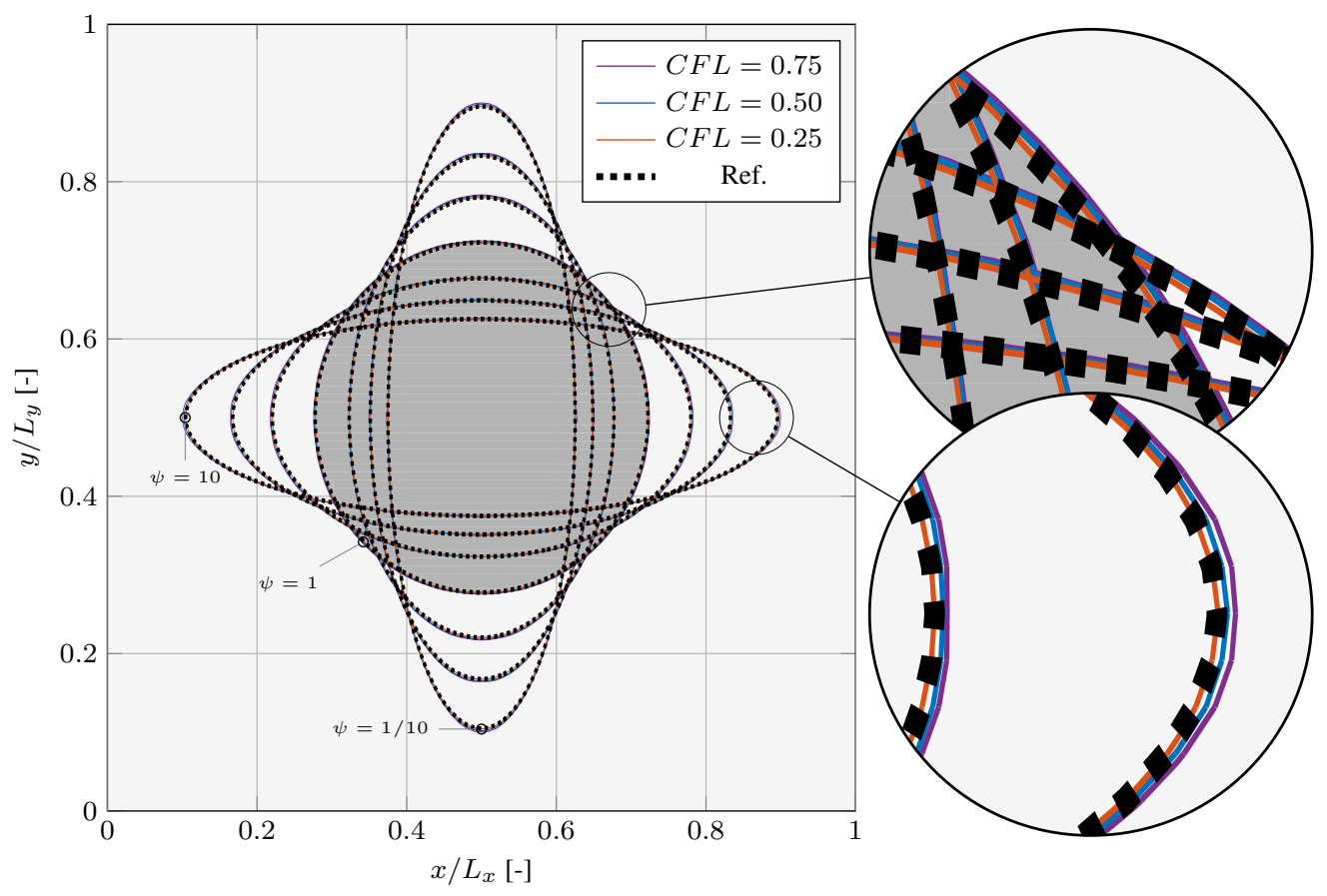

Figure 5: Referring to Fig. 3(a), this figure illustrates the flow front positions after a volume of $S / w_{z}=0.2\left[\mathrm{~m}^{2}\right]$ has been injected. The flow fronts are shown for different degrees of anisotropy, $\psi=K_{1} / K_{2}=(10,5,2.5,1,1 / 2.5,1 / 5,1 / 10)$ and is compared to the analytical solution (ref.) for different time step sizes, $\Delta t$. The filled contour illustrates the resin domain for the isotropic case, $\psi=1$.

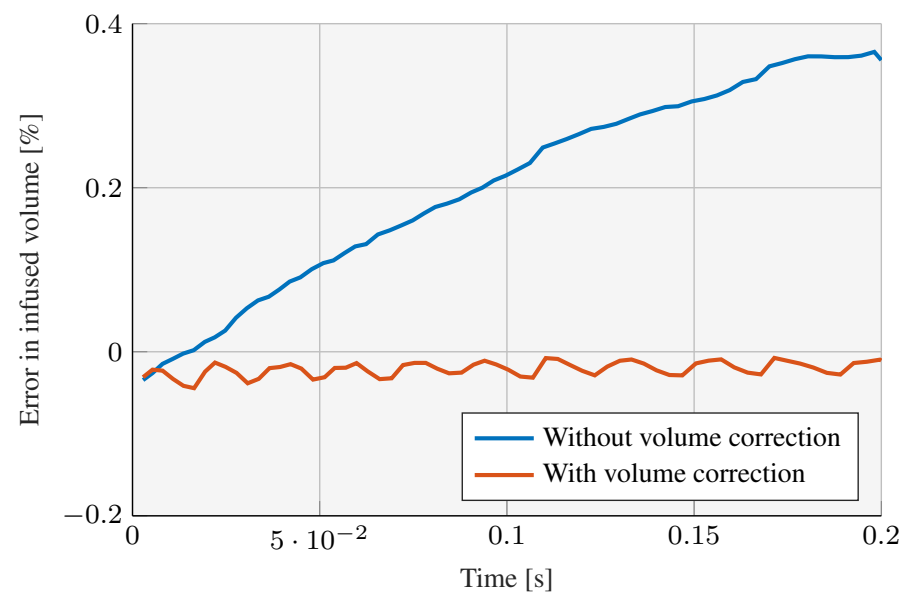

Figure 6: The error of the infused volume for the example in Sec. 3.1.1 with and without the application of the volume correction scheme. 
(a) Flow front position

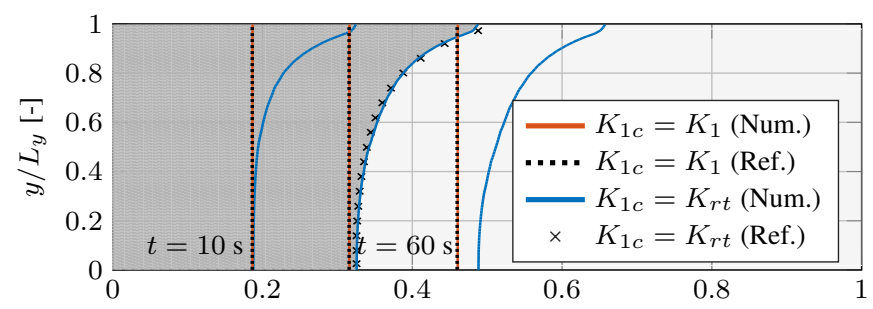

(b) Pressure
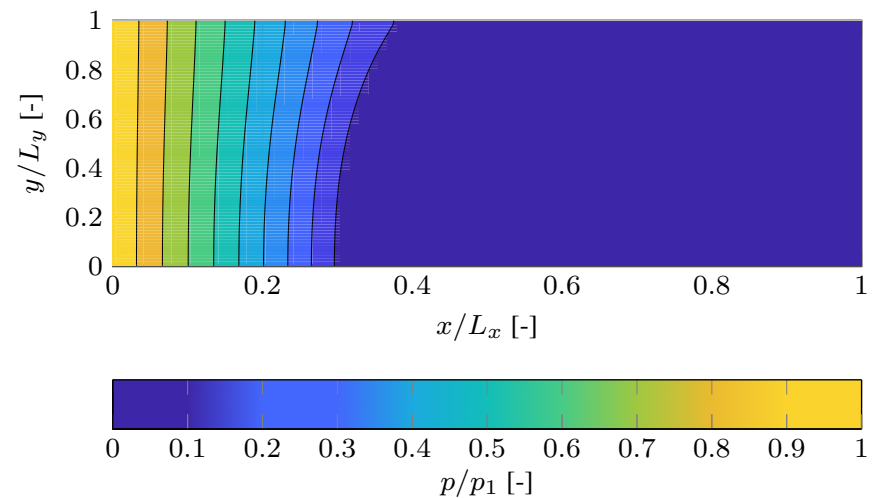

Figure 7: Referring to Fig. 3(b), (a) illustrates the flow front after $t=10,28.4$ and $60 \mathrm{~s} . K_{1 c}=K_{1}$ is for a homogeneous fabric, where the flow front location can be compared to an analytical solution. $K_{1 c}=K_{r t}$ is when a highly permeable race-track near the mould edge is present. For this case, the solution is compared to Yang et al. [41], who revisits the experiments by Young [42]. (b) shows the pressure field at $t=28.4 \mathrm{~s}$ for $K_{1 c}=K_{r t}$. 


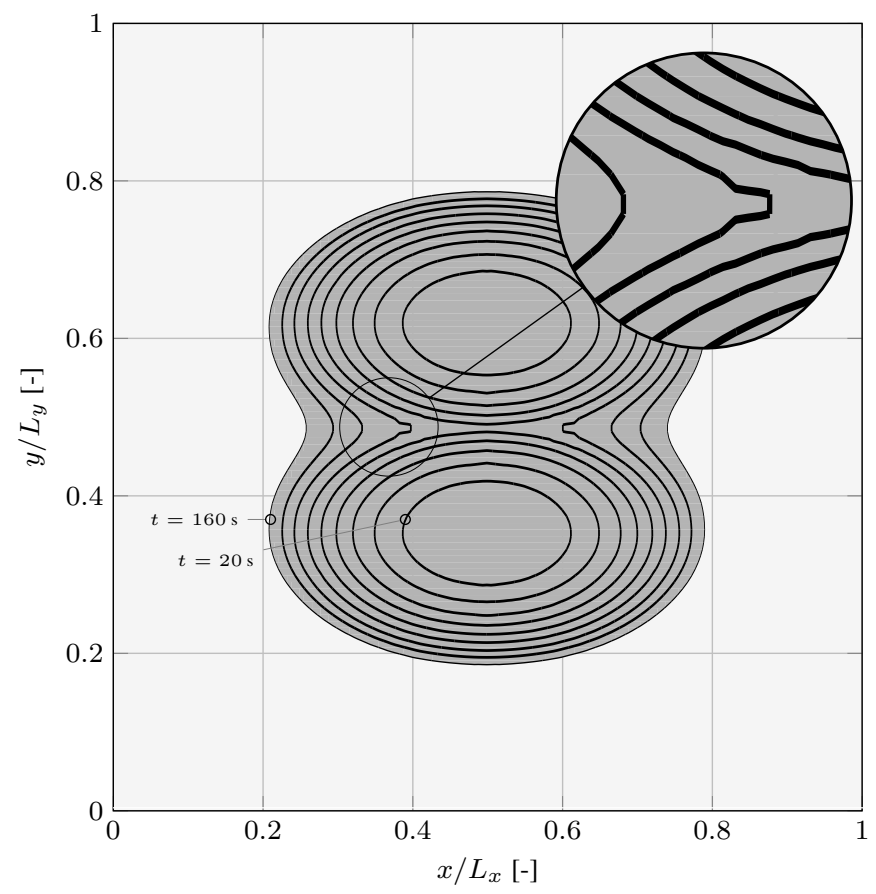

Figure 8: Referring to Fig. 3(c), the figure illustrates flow front positions in increments of $20 \mathrm{~s}$. The filled contour is the resin domain after $160 \mathrm{~s}$.

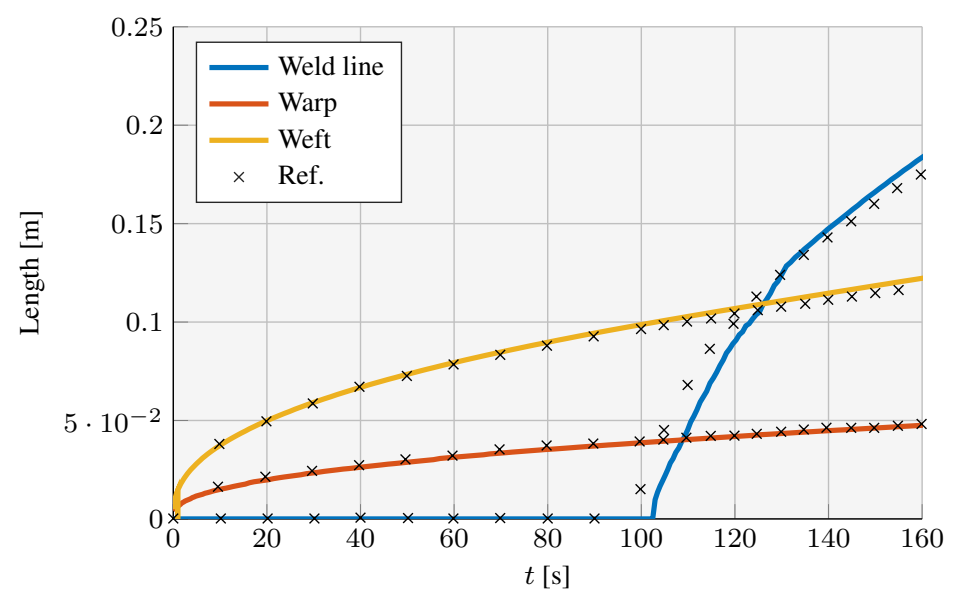

Figure 9: Referring to Fig. 3(c), the figure illustrates the development of the flow front in the warp and weft direction, together with the weld line. (ref.) are experimental results from Shimada et al. [33] 


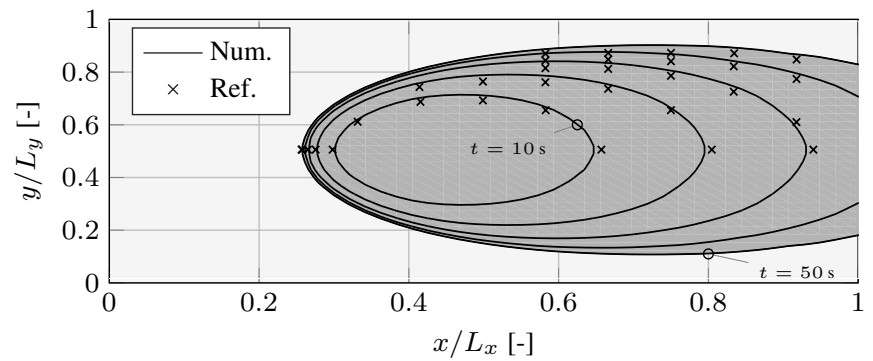

Figure 10: Resin-injection pultrusion (Fig. 3(d)). The flow front is shown for time-increments of $10 \mathrm{~s}$ and is compared to numerical results from Liu [22] (ref.). The filled contour corresponds to the resin domain after $50 \mathrm{~s}$.

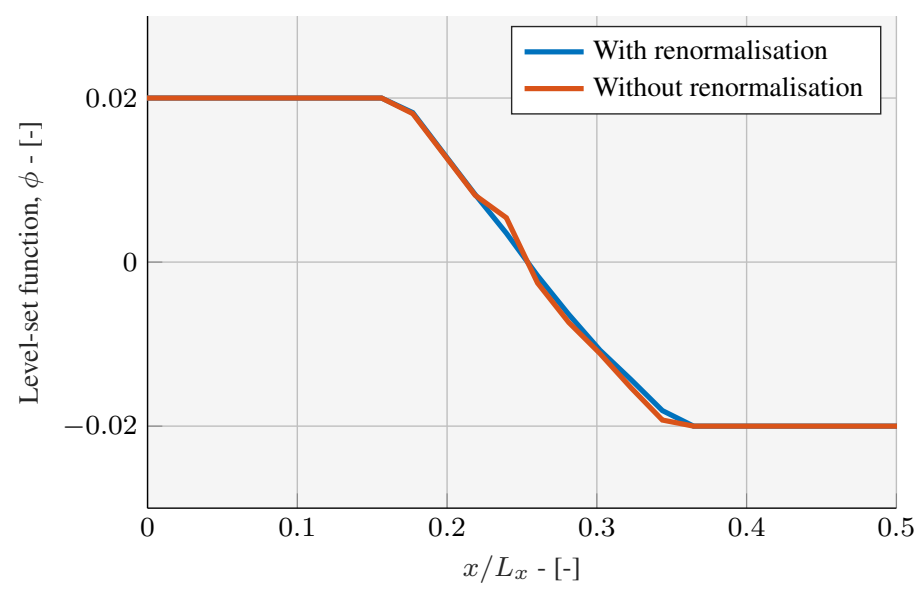

Figure 11: The level-set function at the centre line for resin-injection pultrusion at steady state with and without the application of renormalisation (cf. Sec. 2.2.6). While the interface location is the same (the intersection with the $x$-axis), the level-set function becomes steep in the vicinity of the interface when the renormalisation is not applied for this particular example. 


\section{List of Tables}

Parameters used in the case studies.

*The letters refer to Fig. 3.

$* * \theta_{v}$ and $\mu$ are set to unity, as the fraction, $K /\left(\theta_{v} \mu\right)$, is calculated based on Shimada et al. [33]

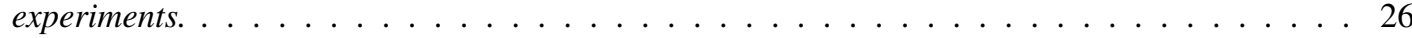




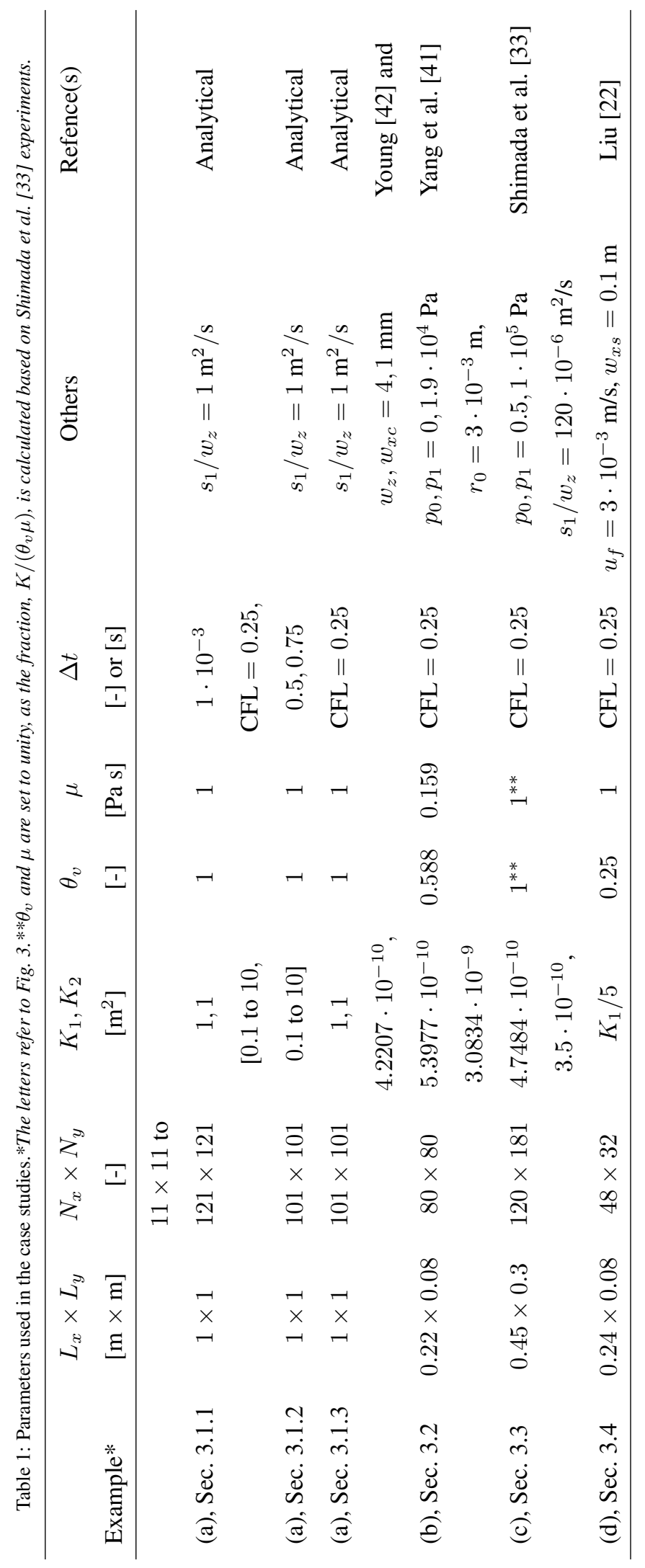

